\title{
Structural differences between dengue viruses circulating in humans and viruses used for vaccine research
}

\author{
Rajendra Raut*,1 (iD) \& Aravinda M de Silva ${ }^{1}$ \\ ${ }^{1}$ Department of Microbiology \& Immunology, University of North Carolina School of Medicine, Chapel Hill, NC 27599, USA \\ *Author for correspondence: rajendra_raut@med.unc.edu \\ "By using fully mature infectious stocks of low passage, contemporary clinical strains each DENV
serotype, the PRNT can be developed into a better tool for evaluating vaccine responses"
}

First draft submitted: 10 May 2019; Accepted for publication: 21 May 2019; Published online:

8 July 2019

Keywords: cell culture $\bullet$ dengue virus $\bullet$ maturation $\bullet$ neutralization $\bullet$ Sri Lanka $\bullet$ vaccine-prophylactic

Four dengue virus (DENV) serotypes are mosquito-borne flaviviruses of humans. The main mosquito vector of DENV, Aedes aegypti, is difficult to control because urbanization and other human activities create environments that strongly favor this species. Given the challenges of mosquito vector control and the absence of other effective countermeasures, DENVs are thriving and infect several hundred million individuals, living in tropical and subtropical regions of the world [1,2]. Highly effective vaccines have been developed against related flaviviruses such as yellow fever (YFV), Japanese encephalitis (JEV) and tick-borne encephalitis (TBE) viruses [3]. The development of DENV vaccines is more challenging because of the presence of four serotypes and the possibility of inadequate immunity enhancing the replication of DENVs and thus, increasing the risk of severe disease [3]. Three live attenuated tetravalent DENV vaccines are at different stages of testing in people. Most recently, the public health community and vaccine developers faced a major setback when the leading candidate, Dengvaxia, was discovered to have a safety signal in some children [4]. Furthermore, we recently discovered that dengue serotype 1 viruses (DENV1) widely used for laboratory research are structurally different from DENV1 viruses circulating in the plasma of those infected [5]. Here, we discuss our findings and the implications for DENV research and vaccines.

Starting in the 1960 s, cell culture-based viral plaque reduction neutralization tests (PRNT) have been used as a gold standard to detect human antibodies correlated with protective immunity to flaviviruses. A PRNT 50 titer (serum dilution neutralizing 50\% of infectious virus) equal to or greater than 1:10 has been established clinically as protective for YFV and JEV [6,7]. Although a PRNT $_{50}$ threshold titer has not been established for each DENV serotype, regulatory agencies and vaccine developers have relied on the presence of neutralizing antibodies to guide the development of DENV vaccines [8]. However in dengue vaccine clinical trials, many individuals with neutralizing antibodies were not protected indicating that, unlike in the case of JEV and YFV, the mere presence of neutralizing antibodies was not sufficient for protection from DENVs [9]. As described next, structural differences between DENVs produced in the laboratory and DENVs circulating in people, may explain why some people who received DENV vaccines experienced breakthrough infections despite developing neutralizing antibodies.

\section{Maturation state of DENV circulating in people}

DENVs contain two integral membrane proteins, envelope (E) and premembrane (prM), which are embedded within the lipid bilayer derived from host cell endoplasmic reticulum during virion assembly. As assembled immature virions move through the secretory pathway, PrM protein is cleaved by a host furin protease in the trans-Golgi network. The cleavage of prM leads to the rearrangement of $\mathrm{E}$ proteins to form mature infectious virions, which are released from infected cells. It has been widely recognized that prM processing is inefficient in cell lines widely used to produce laboratory stocks of DENVs. DENVs produced in laboratories are a heterogeneous mixture of immature, partially mature and fully mature virions containing variable amounts of prM [10]. Using conventional 
assays, it has been challenging to determine the structure, composition and maturation state of DENVs circulating in people because of the transient, low viremia and the large excess of other plasma proteins.

Recently, we characterized the maturation state of DENV1 in human plasma using samples from an epidemic in Sri Lanka and a novel immunocapture assay to detect virions with unprocessed prM protein on their surface [5]. For this study, we only used blood samples from people experiencing primary infections collected before the development of specific antibodies (first 4 days of symptoms), which are likely to interfere with our assays. We observed that DENV1 in the plasma of infected patients had very low or no detectable prM protein on the surface indicating that the virions were fully mature. In contrast, when the same plasma DENVs were passaged once or twice in mammalian or insect laboratory cell lines, most virions released from the cells contained prM protein on the surface indicating incomplete processing and maturation. Laboratory cell lines selected for growing DENVs support robust viral replication. It is likely that the high concentration of intracellular virions overwhelms the host furin protease in the trans-Golgi apparatus that cleaves prM to generate mature virions. In support of this model, we observed that Vero cells engineered to overexpress furin released DENV1 that were more mature than DENV1 released from regular Vero cells [5].

Our studies also demonstrated that plasma DENV1 had a 10- to 100-fold greater specific infectivity compared with DENV1 clinical and laboratory strains produced using laboratory cell lines. The difference in specific infectivity could not be completely attributed to the differences in maturation state indicating there are other structural and biochemical differences between plasma and cell culture viruses beyond maturation state.

\section{Implications for vaccine development}

The PRNT, which utilizes cell lines and laboratory produced stocks of DENVs, has been a workhorse for measuring neutralizing antibody and for guiding the development of dengue vaccines. We performed PRNTs to compare the ability of human antibodies to neutralize human plasma and cell culture-derived DENV1. DENV1 serotype specific human monoclonal antibodies (mAbs) and homotypic immune sera from people exposed to primary DENV1 infections were effective at neutralizing both plasma and cell culture produced DENV1 [5]. DENV serotype cross-reactive human monoclonal antibodies (mAbs) and heterotypic immune sera from people exposed to primary DENV2 or DENV3 infections efficiently neutralized cell culture produced DENV1 and poorly neutralized or failed to neutralize human plasma derived DENV1. Other studies have also demonstrated that DENVs released from primary human cells [11] and mature DENV from furin overexpressing cells [12,13] are resistant to neutralization by some cross-reactive antibodies compared with immature virions produced using standard cell lines. Most human DENV serotype-cross reactive antibodies target conserved epitopes at or near the fusion loop at the tip of domain II of $\mathrm{E}$ protein. This region of $\mathrm{E}$ protein is partially exposed and accessible to antibody binding in immature virions with unprocessed prM, whereas in mature virions the fusion loop is buried and not readily accessible to antibody binding [14]. Our findings suggest that the current PRNT utilizing partially mature DENV stocks will overestimate the levels of DENV neutralizing antibodies induced by vaccines. Indeed, many people who received a leading dengue vaccine candidate experienced breakthrough infections despite having relatively high PRNT50 titers after vaccination [9]. We need to re-examine ways of growing DENVs in cell culture to better reflect the properties of viruses circulating in people. By using fully mature infectious stocks of low passage, contemporary clinical strains each DENV serotype, the PRNT can be developed into a better tool for evaluating vaccine responses.

\section{Financial and competing interests disclosures}

This work was supported by National Institute of Allergy and Infectious Diseases Grants 1-R01-Al107731-01 (primary investigator AM de Silva, UNC) and P01 Al106695 (primary investigator E Harris, University of California at Berkeley). The authors have no other relevant affiliations or financial involvement with any organization or entity with a financial interest in or financial conflict with the subject matter or materials discussed in the manuscript apart from those disclosed.

No writing assistance was utilized in the production of this manuscript.

\section{References}

1. Bhatt S, Gething PW, Brady OJ et al. The global distribution and burden of dengue. Nature 496(7446), 504-507 (2013).

2. Guzman MG, Halstead SB, Artsob H et al. Dengue: a continuing global threat. Nat. Rev. Microbiol. 8(12 Suppl.), S7-S16 (2010).

3. Heinz FX, Stiasny K. Flaviviruses and flavivirus vaccines. Vaccine 30(29), 4301-4306 (2012).

4. Sridhar S, Luedtke A, Langevin E et al. Effect of dengue serostatus on dengue vaccine safety and efficacy. N. Engl. J. Med. 379(4), 327-340 (2018). 
5. Raut R, Corbett KS, Tennekoon RN et al. Dengue type 1 viruses circulating in humans are highly infectious and poorly neutralized by human antibodies. Proc. Natl Acad. Sci. USA 116(1), 227-232 (2019).

6. Hombach J, Solomon T, Kurane I, Jacobson J, Wood D. Report on a WHO consultation on immunological endpoints for evaluation of new Japanese encephalitis vaccines, WHO, Geneva, 2-3 September, 2004. Vaccine 23(45), 5205-5211 (2005).

7. Monath TP. Yellow fever vaccine. Expert Rev. Vaccines 4(4), 553-574 (2005).

8. Roehrig JT, Hombach J, Barrett AD. Guidelines for Plaque-reduction neutralization testing of human antibodies to dengue viruses. Viral Immunol. 21(2), 123-132 (2008).

9. Moodie Z, Juraska M, Huang Y et al. Neutralizing antibody correlates analysis of tetravalent dengue vaccine efficacy trials in Asia and Latin America. J. Infect. Dis. 217(5), 742-753 (2018).

10. Lok SM. The interplay of dengue virus morphological diversity and human antibodies. Trends Microbiol. 24(4), 284-293 (2016).

11. Dejnirattisai W, Wongwiwat W, Supasa $\mathrm{S}$ et al. A new class of highly potent, broadly neutralizing antibodies isolated from viremic patients infected with dengue virus. Nat. Immunol. 16(2), 170-177 (2015).

12. Tsai WY, Chen HL, Tsai JJ et al. Potent neutralizing human monoclonal antibodies preferentially target mature dengue virus particles: implication for novel strategy for Dengue vaccine. J. Virol. 92(23), pii:e00556-18 (2018).

13. Mukherjee S, Sirohi D, Dowd KA et al. Enhancing dengue virus maturation using a stable furin over-expressing cell line. Virology 497 , 33-40 (2016).

14. Cherrier MV, Kaufmann B, Nybakken GE et al. Structural basis for the preferential recognition of immature flaviviruses by a fusion-loop antibody. EMBO J. 28(20), 3269-3276 (2009). 